\title{
Efectividad del programa educativo "Mi amigo y yo, unidos contra la TB" en la prevención de tuberculosis a estudiantes de una institución educativa pública, Ate- Vitarte, Lima
}

\author{
Effectiveness of the educational program "My friend and I, united against TB" in the \\ prevention of tuberculosis to students of a public educational institution, Ate-Vitarte, \\ Lima
}

Yanela Ricalde Castillo', Milagros Celis Mendoza², Janeth Chávez Sosa ${ }^{3}$

\section{RESUMEN}

Objetivo: Determinar la efectividad del programa educativo "Mi amigo y yo, unidos contra la TB" en los conocimientos y actitudes para la prevención de tuberculosis en los estudiantes del segundo grado de una institución educativa pública en Ate-Vitarte. Materiales y métodos: el trabajo fue de enfoque cuantitativo, diseño cuasi-experimental y de corte longitudinal, la muestra estuvo conformada por 102 estudiantes de 12 a 15 años, 51 del grupo control y 51 del grupo experimental. El instrumento empleado fue el cuestionario, además de la creación del módulo del programa que constó de 9 unidades abordadas en 24 sesiones educativas, con una duración de 5 meses. Resultados: el programa fue efectivo en los conocimientos y actitudes sobre la prevención de TB con un p-valor $<0,05$. Conclusión: la intervención educativa fue efectiva para la prevención de dicha enfermedad.

Palabras clave: Prevención sobre la tuberculosis, conocimientos y actitudes.

\section{ABSTRACT}

Objective: To determine the effectiveness of the educational program "My friend and I, united against TB" in the knowledge and attitudes for the prevention of tuberculosis in the second grade students of a public educational institution in Ate-Vitarte. Materials and methods: the work was quantitative, quasiexperimental design and longitudinal cut, the sample consisted of 102 students from 12 to 15 years, 51 of the control group and 51 of the experimental group. The instrument used was the questionnaire, in addition to the creation of the program module that consisted of 9 units addressed in 24 educational sessions, with a duration of 5 months. Results: the program was effective in knowledge and attitudes about the prevention of TB with a p-value $<0.05$. Conclussion: the educational intervention was effective for the prevention of said disease.

Keywords: Prevention of tuberculosis, knowledge and attitudes. 


\section{INTRODUCCIÓN}

Wilches, Hernández, Hernández y Pérez (2016) mencionan que la tuberculosis (TB) es una enfermedad prevenible y curable, sin embargo, en el mundo sigue siendo un problema de salud pública, debido a la estrecha relación con los factores sociales y ambientales que aumentan el riesgo de contraer dicha enfermedad. En el mundo, se estima que hay unos 9,4 millones de casos nuevos de TB y 1,7 millones de muertes que ocurren cada año por esta causa. Asimismo, esta enfermedad es considerada como una de

las enfermedades infectocontagiosas de mayor prevalencia en países en vías de desarrollo, siendo la segunda causa principal de muerte (Organización Panamericana de la salud, 2016).

Asimismo, en estudios epidemiológicos realizados en el 2015, reportaron que el mayor número de los casos de tuberculosis ocurrieron en Asia $(61 \%)$ y la región africana $(26 \%)$. A su vez, las proporciones fueron menores en la región mediterránea del Este $(7 \%)$, la región europea $(3 \%)$ y la región de las américas $(3 \%)$ (Plataforma regional Americana Latina y el Caribe, 2016).

A nivel de Latino América, la Organización Panamericana de la Salud (OPS) indica que, en las américas 268.500 personas se enfermaron de TB y 18.500 murieron por dicho mal. Es así que, en América Latina y el Caribe existen más de 230000 casos anuales de individuos diagnosticados con tuberculosis; sin embargo, se estima que en realidad hay cerca de 400 000 casos, lo que revela un sub registro por encima del $40 \%$ de casos de TB. (Organización Panamericana de la salud, 2016).

Según el diario El Comercio, el Perú tiene, después de Haití, la tasa más alta de tuberculosis de Latinoamérica. En el último año se contabilizaron 33 mil casos de tuberculosis sensible (la enfermedad en primer grado), de los cuales el $59 \%$ se presenta en Lima (Subirana, 2011).

De igual manera, Alarcón, Alarcón, Figueroa y Mendoza (2015) refieren que, el $80 \%$ de los casos, en el año 2015 fueron reportados por diez regiones de salud, siendo Lima Metropolitana (Lima Este, Lima Ciudad y Lima Sur) la ocupa en primer lugar las estadísticas seguidos de el Callao, La Libertad, Loreto, Ica, Lima Provincias,
Junín, Lambayeque, Arequipa y Ucayali. Lima Metropolitana y la Región Callao notificaron el $59,3 \%$ de todos los casos de TB a nivel nacional en el año 2015.

Así mismo El diario el Comercio menciona que cada diez pacientes con este mal radican en Lima, sobre todo en áreas tugurizadas de siete distritos: San Juan de Lurigancho, Rímac, La Victoria, El Agustino, Ate, Santa Anita y Barranco. La variedad predominante en la capital es la sensible, cuya curación tarda siete meses, si no se interrumpe el tratamiento (Sandoval, 2016).

Por otro lado, en el Perú, las estadísticas en adolecentes del Ministerio de Salud, registraron en el 2010 alrededor de 32,477 casos de tuberculosis; De este total, el 2,8\% afectó a niños entre 0 a 9 años, el $3.57 \%$ a niños y adolescentes entre 10 y 14 años y el $13.43 \%$ a adolescentes entre los 15 y 19 años de edad. Por tanto, se deduce que el $20 \%$ de los afectados (6,496 casos) fueron niños y adolescentes (Choque, 2011).

Asimismo, la incidencia de TB en los menores de 15 años se ha mantenido estacionaria en los últimos 5 años con un promedio de 30 niños afectados por TB por cada 100 mil niños menores de 15 años. El porcentaje de casos menores de 15 años que tuvieron contacto con otros pacientes con TB, fue mayor (49.2\%) que los mayores de 15 años (30\%); además, este porcentaje se incrementó en menores de 5 años (63\%) (Soto y Chavez, 2015).

Por su parte Gutiérrez, Moreno y Gonzáles (2010) menciona que todo niño que haya tenido contacto con un enfermo de tuberculosis con baciloscopia positiva, debe ser considerado como enfermo o como contacto según corresponda.

Según, la Organización Mundial de la Salud menciona que el riesgo de infección se basa no sólo en las probabilidades de estar en contacto con el bacilo, sino también, en el nivel de comprensión y aplicación de medidas preventivas (Wilches et al., 2016).

Por otro lado, en la entrevista personal realizada a la Jefa de la estrategia de PCT (programa de control de tuberculosis) del Centro de Salud San Fernando refirió que después de realizar un seguimiento y detección de casos sintomáticos respiratorios a la población perteneciente 
al centro de salud, encontró que el $40 \%$ de los pobladores son posibles sintomáticos respiratorios, de los cuales la mayoría nunca se ha realizado una prueba de baciloscopia y no tienen la intención de hacerlo. También refirió que la mayoría de pobladores que están bajo la jurisdicción del centro de salud tienen familiares cercanos que estudian en la institución educativa José Abelardo Quiñones lo que es un riesgo latente para la población estudiantil.

Así mismo después de realizar una entrevista personal con el Sub-Director de la institución educativa José Abelardo Quiñones N. ${ }^{\circ} 1138$ "refirió que en el año 2016 se encontraron 2 casos confirmados de tuberculosis pulmonar en los estudiantes de primer año de secundaria y que el $30 \%$ de alumnos en el nivel secundario tuvo deserción escolar después de considerarlos como posibles sintomáticos respiratorios.

En relación a la prevención Villar (2011) menciona que el sistema de salud ha tenido modificaciones importantes orientadas al fortalecimiento de la Atención Integral de la Salud, fundamentadas en la necesidad de fortalecer la atención en el nivel primario de atención. El objetivo de la atención de la salud es la promoción de la salud y a la prevención de la enfermedad, dando relevancia al primer nivel de atención y a las acciones dirigidas a la prevención.

Desde un enfoque sociológico, Contreras, Contreras, Jiménez, Solís y Torres (2004) señala que: "la Salud Pública lo considera como el mejor ejemplo de enfermedad social, por estar relacionada a la pobreza, malnutrición, factores culturales, estilos de vida no saludables, falta de atención primaria, así como de programas de difusión y prevención". De manera similar el Ministerio de Salud (2010) indicó que las personas más vulnerables son aquellas que reúnen la mayor parte de factores de riesgo, más aún si estas personas sufren de hacinamiento habitacional y destruición lo que genera un compromiso grande para el contagio de la tuberculosis.

\section{MATERIAL Y MÉTODOS}

El estudio corresponde a un enfoque cuantitativo, diseño cuasi- experimental, de corte longitudinal, en un grupo control y experimental, en los estudiantes de segundo año de secundaria. La muestra estuvo conformada por 102 estudiantes entre 12 a 15 años de edad de ambos grupos, el muestreo muestra fue no probabilístico de tipo intencional empleando criterios de inclusión y exclusión, contando con 51 alumnos participantes del programa. El cuestionario de conocimiento y actitudes fue adaptado por las investigadoras, este fue sometido a una prueba piloto 54 estudiantes.

La fiabilidad del cuestionario de conocimientos en tuberculosis, fue Kunder - Richardson 20. La confiabilidad tipo consistencia interna se refiere al grado en que los ítems de una escala se correlacionan entre ellos. La consistencia interna se calcula con la fórmula 20 de Kunder - Richardson para las escalas dicotómicas. La consistencia interna se considera aceptable cuando se encuentra entre 0,70 y 0,90 (Campo Oviedo, 2008) El KR20 obtenido fue 0,743.

Se utilizó la metodología del programa 'Mi amigo y yo unidos contra la TB" constó de 9 unidades abordadas en 24 sesiones educativas teóricas y prácticas, cada sesión de clase tenía una duración de 30 minutos realizadas dos veces por semana en el curso de tutoría durante los meses mayo / setiembre del 2017. Para su aplicación se emplearon estrategias como el modulo educativo, planes de clase, talleres de demostración y materiales audiovisuales. Los datos encontrados se analizaron con la prueba no paramétrica Wilcoxon. Cuya prueba se basa en comparar el rango medio de dos muestras relacionadas y determinar si existen diferencias entre ellas, el estudio fue muy significativo con un $\mathrm{p}$-valor 0,0001 . 
Efectividad del programa educativo "Mi amigo y yo, unidos contra la TB" en la prevención de tuberculosis a estudiantes de una institución educativa pública, Ate- Vitarte, Lima

\section{RESULTADOS}

Tabla 1

Datos sociodemográficos de los tutores del grupo control y experimental de los estudiantes del segundo grado de secundaria de una institución educativa pública, Ate- Vitarte, 2017.

\begin{tabular}{|c|c|c|c|c|}
\hline \multirow{2}{*}{ Grupo Variable } & \multicolumn{2}{|c|}{ Experimental } & \multicolumn{2}{|c|}{ Control } \\
\hline & $\mathbf{N}$ & $\%$ & $\mathbf{N}$ & $\%$ \\
\hline \multicolumn{5}{|c|}{ Antecedentes de tuberculosis } \\
\hline Si & 13 & 25,5 & 12 & 23,5 \\
\hline No & 38 & 74,5 & 39 & 76,5 \\
\hline \multicolumn{5}{|l|}{ Tipo de vivienda } \\
\hline Adobe & 2 & 3,9 & 1 & 2,0 \\
\hline Material noble & 27 & 52,9 & 17 & 33,3 \\
\hline Pre fabricada & 7 & 13,7 & 8 & 15,7 \\
\hline Otros & 15 & 29,4 & 25 & 49,0 \\
\hline \multicolumn{5}{|l|}{ Nivel de instrucción } \\
\hline Sin estudios & 1 & 2,0 & 1 & 2,0 \\
\hline Estudios Primarios & 15 & 29,4 & 11 & 21,6 \\
\hline Estudios secundarios & 30 & 58,8 & 31 & 60,8 \\
\hline Estudios técnicos & 3 & 5,9 & 4 & 7,8 \\
\hline Estudios universitarios & 2 & 3,9 & 4 & 7,8 \\
\hline \multicolumn{5}{|l|}{ Profesión del Tutor } \\
\hline Empleado Público & 8 & 15,7 & 9 & 17,6 \\
\hline Trabajador Independiente & 43 & 84,3 & 42 & 82,4 \\
\hline \multicolumn{5}{|l|}{ Ingreso mensual } \\
\hline$>750$ & 30 & 58,8 & 27 & 52,9 \\
\hline $750-1500$ & 17 & 33,3 & 21 & 41,2 \\
\hline $1500-2500$ & 4 & 7,8 & 3 & 5,9 \\
\hline \multicolumn{5}{|l|}{ Número de hijos } \\
\hline 1 & 7 & 13,7 & 3 & 5,9 \\
\hline 2 & 15 & 29,4 & 19 & 37,3 \\
\hline 3 & 7 & 13,7 & 5 & 9,8 \\
\hline 4 & 7 & 13,7 & 9 & 17,6 \\
\hline 5 & 15 & 29,4 & 15 & 29,4 \\
\hline \multicolumn{5}{|l|}{ Tipo de familia } \\
\hline Nuclear & 28 & 54,9 & 29 & 56,9 \\
\hline Monoparental & 7 & 13,7 & 4 & 7,8 \\
\hline Extensa & 16 & 31,4 & 18 & 35,3 \\
\hline \multicolumn{5}{|l|}{ Número de Ventanas } \\
\hline 1 & 14 & 27,5 & 13 & 25,5 \\
\hline 2 & 18 & 35,3 & 21 & 41,2 \\
\hline 4 & 19 & 37,3 & 17 & 33,3 \\
\hline \multicolumn{5}{|l|}{ Número de habitaciones } \\
\hline 1 & 7 & 13,7 & 10 & 19,6 \\
\hline 2 & 12 & 23,5 & 15 & 29,4 \\
\hline 3 & 12 & 23,5 & 7 & 13,7 \\
\hline
\end{tabular}




\begin{tabular}{lcccc}
4 & 13 & 25,5 & 12 & 23,5 \\
Más de 4 & 7 & 13,7 & 7 & 13,7 \\
$\begin{array}{l}\text { Número de personas que } \\
\text { duermen en cada habitación }\end{array}$ & & & \\
1 & 11 & 21,6 & 4 & 7,8 \\
2 & 28 & 54,9 & 29 & 56,9 \\
3 & 6 & 11,8 & 7 & 13,7 \\
4 & 1 & 2,0 & 5 & 9,8 \\
Más de 4 & 5 & 9,8 & 6 & 11,8 \\
Total & 51 & 100 & 51 & 100 \\
\hline
\end{tabular}

Tabla 2

Diferencias del nivel de conocimientos y actitudes, antes y después del programa "Mi amigo y yo, unidos contra la TB" del grupo experimental en los estudiantes del segundo grado de secundaria de una institución educativa pública, según la prueba estadística Wilcoxon.

\begin{tabular}{|c|c|c|c|c|c|c|}
\hline Grupo & Variable & $\mathbf{n}$ & Media & ds & $\mathbf{z}$ & $\mathbf{P}$ \\
\hline \multirow{3}{*}{ Experimental } & Conocimiento & & & & \multirow{3}{*}{4,811} & \multirow{3}{*}{0,000} \\
\hline & Pre test & 51 & 7,45 & 2,110 & & \\
\hline & Pos test & 51 & 11,56 & 1,878 & & \\
\hline \multirow{3}{*}{ Experimental } & Actitudes & & & & & \multirow{3}{*}{0,00} \\
\hline & Pre test & 51 & 40,60 & 6,826 & & \\
\hline & Pos test & 51 & 41,84 & 2,773 & & \\
\hline
\end{tabular}

Tabla 3

Nivel de conocimiento sobre prevención de tuberculosis antes y después del programa educativo en los estudiantes del segundo grado de secundaria de una Institución Educativa Pública, Ate- Vitarte, 2017.

\begin{tabular}{clcccc}
\multirow{2}{*}{ Grupo } & Conocimiento & \multicolumn{2}{c}{ Pre test } & \multicolumn{2}{c}{ Post test } \\
\cline { 3 - 5 } Experimental & Deficiente & 5 & 9,8 & 2 & n \\
& Regular & 30 & 58,8 & 0 & 0 \\
& Bueno & 16 & 31,4 & 49 & 96,9 \\
\cline { 2 - 5 } Control & Deficiente & 11 & 21,6 & 15 & 29,4 \\
& Regular & 21 & 41,2 & 20 & 39,2 \\
& Bueno & 19 & 37,3 & 16 & 31,4 \\
\hline
\end{tabular}

Tabla 4

Nivel de actitudes sobre prevención de tuberculosis en los estudiantes del segundo grado de secundaria de una institución educativa pública, Ate- Vitarte, 2017.

\begin{tabular}{llcccc}
\multirow{2}{*}{ Grupo } & Actitudes & \multicolumn{2}{c}{ Pre test } & \multicolumn{2}{c}{ Post test } \\
\cline { 3 - 6 } Experimental & Negativa & 23 & 45,1 & 2 & $\%$ \\
& Positiva & 28 & 54,9 & 49 & 3,9 \\
\multirow{2}{*}{ Control } & Negativa & 21 & 41,2 & 17 & 36,1 \\
& Positiva & 30 & 58,8 & 34 & 66,7 \\
\hline
\end{tabular}




\section{DISCUSIÓN}

La tuberculosis constituye un grave problema de salud pública en el mundo que aún no ha podido ser resuelto en su totalidad, especialmente en países en vías de desarrollo. Su prevalencia oscila entre los 30 millones de casos al año con una letalidad de 2 a 3 millones. Asimismo, se sabe que los adolescentes y adultos mayores constituyen alrededor de la tercera parte del total de casos afectados (Robles, 2013).

Por tanto, la OMS (2014) indica que las intervenciones eficaces durante la adolescencia protegen las inversiones de salud pública en la supervivencia infantil y el desarrollo en la primera infancia, siendo que, la adolescencia ofrece la oportunidad de corregir problemas que hayan podido surgir en los 10 primeros años de vida y así evitar que afecten a la salud en el futuro.

Asimismo, Morales, Ferreira, Oduardo, Pompa, y Areas (2015) mencionan que "los factores de riesgo de la tuberculosis influyen tanto en la posibilidad de exponerse al contagio, como en el desarrollo final de la enfermedad, puesto que una persona infectada no siempre enferma, sino más bien el conjunto de factores de riesgo son los que determinan las probabilidades de su evolución hacia un estado patológico. En adición, en países donde además de la pobreza y la inequidad; la estigmatización, exclusión y discriminación son males sociales, la situación se agudiza y vuelve a los pobres más vulnerables".

Al analizar los datos sociodemográficos ver en la tabla 7 se encontró que la mayoría de niños presentaba una edad entre 12 y 15 años, de los cuales un $25,5 \%$ presentó un antecedente familiar de tuberculosis. Con respecto a esto, Carbajal (2016) resalta que, durante la infancia el riesgo de contagio de tuberculosis es mayor, siendo de un $40 \%$ en niños menores de 1 año, el $25 \%$ de 1 a 5 años, el $15 \%$ de 11 a 15 años y solo un 5 y $10 \%$ en adultos.

De igual manera, Gutiérrez, Gutiérrez y Toledo (2005) resaltan que, es más probable que las personas enfermas con TB contagien a otras personas con las que pasan la mayor parte del tiempo. Esto incluye familiares, amigos y compañeros de trabajo.

En el ámbito social, ver tabla 8 diversos estudios han demostrado que la tuberculosis predomina en personas con una escolaridad baja, ingresos económicos insuficientes y una ocupación laboral artesanal y/o desempleados. Es así, que en este estudio se encontró que la mayoría de los tutores indicó un ingreso mensual menor de 750 soles, con un grado de instrucción de secundaria y una ocupación de independiente (ambulante).

En relación a esto, Lozano, Plasencia, Ramos, García, y Mahíquez (2009) afirman que el nivel escolar es un compone que condiciona la ocupación laboral, la cultura y la educación. Así mismo, la condiciones físicas, químicas y biológicas del puesto de trabajo, puede o no favorecer el desarrollo de malos hábitos. Por último, también señalan que, el ingreso económico es un factor importante, así como su uso y distribución, ya que las personas con un salario estable disponen para alimentarse, vestirse y mejorar su vivienda apropiadamente y destinar una parte de sus gastos para la instrucción de los hijos. La falta extrema de una entrada financiera lleva a la pobreza, problemas de salud todavía y por lo tanto causa un progreso desigual. Entonces se asiente que la tuberculosis está relacionada con un bajo estado socioeconómico, hacinamiento y un menguado nivel de vida que contribuya a trasmitir la infección. Premisa que se corrobora en la investigación, ya que el $25,5 \%$ de los tutores refirieron que viven en hogares con menos de 4 habitaciones y el $54,9 \%$ resaltaron que duermen de dos a más personas en una habitación.

Para Pérez (2007), los programas educativos, en el ámbito escolar, permiten la promoción de hábitos y estilos de vida saludables. Para esto, es necesario el fortalecimiento de conocimientos previos y la identificación de conductas en riesgo. De igual manera, se fundamenta en la participación activa de maestros, padres de familia, personal de salud y autoridades de la comunidad que deberán planear, ejecutar y evaluar las acciones a seguir. Asimismo, los desarrollos de dichos programas en el ámbito educativo permitirán mantener una población cautiva que se encuentra en proceso de formación, lo que favorecerá la sistematización y profundización de conocimientos de acuerdo a las características del desarrollo en cada etapa de la vida, por el impacto que tiene en la sociedad, ya que lo aprendido en la escuela se reproduce y aplica fuera de ella. 
La investigación implementó el programa "Mi amigo y yo, unidos contra la TB" con el propósito de prevenir la tuberculosis en estudiantes del segundo año de secundaria de una institución pública de Ate-Vitarte. Dicho programa se dio en tres fases: La evaluación del impacto, donde se inspeccionó las influencias del programa en el comportamiento de los estudiantes; la evaluación de proceso, en el cual se observó el logro proporcional en relación con los objetivos propuestos por el proyecto; y finalmente, la evaluación de resultados que midió el impacto futuro del programa a través de la solides en los conocimientos, actitudes sobre la prevención de la tuberculosis.

En lo referente a los resultados del estudio, se encontró que existe diferencia significativa en los conocimientos de los estudiantes antes y después del programa educativo con un $p$-valor de 0,0001. De igual manera, los datos descriptivos revelaron que el $58,8 \%$ de los estudiantes presentaron un conocimiento regular antes del programa e indicaron un conocimiento bueno en un $96,9 \%$ después de haber participado en él. Cabe señalar que para el grupo control no se encontró diferencia significativa y permanecieron con un conocimiento regular en un $39,2 \%$.

Resultados similares obtuvieron Crespo, Huapaya y Marca (2014) en su trabajo realizado en Lima: "Respira sano en el nivel de conocimiento sobre la tuberculosis pulmonar en los estudiantes del $3^{\circ}$ al $5^{\circ}$ de secundaria; de la institución educativa corazones marianos del norte", donde el programa resultò altamente significativo con $\mathrm{p}$ - valor de 0.0001 . Del mismo modo, Carta (2013) realizó un estudio en Tacna titulado: "Relación de los conocimientos sobre tuberculosis y las actitudes preventivas de los estudiantes del 4to y 5to grado de nivel secundario de la I.E. Cesar A. Cohaila Tamayo", donde los estudiantes obtuvieron un alto nivel de conocimientos en un $64 \%$.

Bélanger (1997) señala que mejorar los conocimientos ver en la tabla 1 sobre la salud es esencial para mejorar el bienestar y fomentar el desarrollo de la comunidad. De igual forma, quienes están más predispuestos a tener mala salud no son únicamente los más pobres, sino quienes tienen el menor nivel de educación. Es así que, en este estudio se encontró que $58.8 \%$ de los tutores de los adolescentes participantes del programa indicaron un grado de instrucción de secundaria y el $84,3 \%$ presentaban un oficio independiente (ambulantes). Por último, la Unesco resalta que el concepto de la educación para la salud, que actualmente abarca un concepto más amplio de promoción de la salud y un nuevo énfasis en la prevención, se concentra cada vez más en el aprendizaje y en el proceso de fortalecimiento de la autonomía.

En torno a la efectividad de los programas educativos, el MINSA (2004) menciona que, una de las estrategias para prevenir la tuberculosis es a través de la información, educación y comunicación, lo cual permitirá que el individuo adquiera conocimientos sobre este tema. Asimismo, Herrera (2009) menciona que, las estrategias de aprendizaje engloban todo un conjunto de procedimientos y recursos cognitivos que los estudiantes ponen en marcha cuando se enfrentan al aprendizaje; con lo cual, en sentido estricto, se encuentran muy relacionadas con los componentes cognitivos que influyen en el proceso de aprender.

Por otro lado, dentro del campo de la enfermería comunitaria Mendieta, Mena y Mendoza (2012) mencionan que la enfermera debe integrar los conceptos y métodos de la salud comunitaria para promover, proteger, prevenir, mantener y restaurar la salud de la población, así como identificar, por medio del diagnóstico, grupos y conjuntos de individuos que están expuestos a los mismos factores de riesgo y comparten necesidades en salud. Por ende, el rol de la enfermera comunitaria es atender correctamente las necesidades del individuo, grupo y comunidad.

Vincezi y Tedesco (2009) Consideran que la educación y su relación con el mejoramiento de la calidad de vida implica asumir la responsabilidad de promover, tanto desde ámbitos de educación formal como no formal. Por lo tanto, la educación es una condición necesaria para promover la salud de los individuos. La promoción de la salud, desde la intervención educativa, se relaciona con el fortalecimiento de aquellos factores que mejoren la calidad de vida. La educación en su ámbito formal de desarrollo, asume, a través de la escuela, un rol fundamental en la promoción de factores protectores de los individuos. En este sentido, las acciones de promoción y prevención escolar están destinadas a los educandos, a sus familias y a la comunidad circundante.

Otro dato hallado en el estudio indicó que existe diferencia significativa en las actitudes de los 

estudiantes de una institución educativa pública, Ate- Vitarte, Lima

alumnos antes y después del programa "mi amigo y yo, unidos contra la TB" con un p-valor de 0,0001 . De igual manera, los datos descriptivos revelaron que el $96,1 \%$ de los estudiantes pertenecientes al grupo experimental manifestaron una actitud positiva frente a la prevención de tuberculosis a diferencia del grupo control, en la que solo el $66,7 \%$ mantuvo dicha actitud.

Así mismo, Lima, Neves y Lima (2012) realizaron un trabajo en España titulado "Actitudes de adolescentes hacia la salud: evaluación de un programa escolar de promoción de la salud", donde pudieron observar cambios de actitud ver la tabla 2 favorables hacia el comportamiento saludable con un $p$ valor de 0,001 . Por otro lado, Factor (2013) realizò un trabajo en la cuidad de Tacna titulado: "Relación entre el nivel de conocimiento y actitud sobre medidas preventivas frente a la tuberculosis pulmonar en escolares del nivel segundario de la institución educativa Mánuel A. Odria", en el cual se observó que los adolescentes presentaron una actitud positiva en un 70,6 \%.

Fuentes (2009) refiere que el proceso de cambio conductual es un proceso complejo que implica no solo aprender algo nuevo, sino olvidarse de algo que está muy arraigado en la personalidad y en las relaciones sociales del individuo. En este contexto se debe considerar relevante diseñar programas de intervención que consideren la compleja dinámica en su totalidad, diseñando no solo actividades que fomenten el cambio a nivel cognitivo, sino también, considerando la interrelación sujeto- comportamiento-situación y la relación existente entre los componentes actitudinales cognitivo-afectivo- conductual presentes en cada individuo que favorecerán el cambio.

La UNICEF (2002) menciona que casi la mitad de las conexiones cerebrales estaban ya establecidas cuando un niño entraba en el jardín de infancia, y que la única tarea de desarrollo que quedaba era asegurar esas conexiones. Ahora disponemos de estudios recientes que muestran que el cerebro experimenta un ciclo continuo de crecimiento cada pocos años y que, a partir de aproximadamente los 11 años de edad, se produce una explosión de actividad eléctrica y fisiológica, que reorganiza drásticamente miles de millones de redes neuronales que afectan a las aptitudes emocionales y a las habilidades físicas y mentales.
Por otro lado, Oblitas (2013) menciona que todos desean estilos de vida que llevan a la salud, añadir vida a los años, añadir salud a la vida, motivaciones para adquirir y mantener la salud, el incremento de hábitos saludables y el decremento de conductas de riesgo, así como aumentar la productividad y mejorar la calidad de vida. Esto presupone la instauración y mantenimiento de comportamientos saludables y además potenciadores de las capacidades funcionales, físicas, psicológicas y sociales de la persona. La mayoría de las diez principales causas de muerte en los países desarrollados se encuentran determinadas en gran parte por factores de conducta. En consecuencia, podrían reducirse si las personas mejorarán tan solo cinco comportamientos: dieta, hábito de fumar, ejercicio físico, abuso del alcohol, uso de fármacos hipotensores, de los que se hablará más adelante. De igual manera, Risueño (2010) menciona que tener un estilo de vida saludable está basado en las elecciones de las personas de acuerdo a sus costumbres y cultura. Así mismo el estilo de vida está relacionado con la conducta y el carácter consistente, que es congruente con la situación social de una persona y sus condiciones de vida.

Como podemos ver, la tuberculosis es uno de los problemas más graves del momento actual, y a la vez uno de los que menos atención ha recibido en las últimas décadas. Sin embargo, uno de los aspectos más importantes y fascinantes, en relación a este mal, es que es una enfermedad $100 \%$ curable y prevenible, que depende de los determinantes sociales y de salud (Bonilla, 2008).

Finalmente, si se quiere ganar la batalla contra esta enfermedad infecciosa (o contra cualquier otra), no solo se deben dirigir los esfuerzos a la detección y tratamiento del paciente enfermo, sino que es también de importancia vital, que nos esforcemos en evitar que el individuo susceptible pueda enfermar; o sea, que la prevención debe ocupar un lugar relevante en cualquier programa antituberculoso que se quiera emprender (Farga, 2006).

\section{Declaración de financiamiento y de conflictos de interés:}

El estudio fue financiado por los autores, quienes declaran no tener conflictos de interés. 


\section{Correspondencia}

Yanela Karoly Ricalde Castillo

Correo electrónico: yanelarc@upeu.edu.pe

Milagros Celis Mendoza

Correo electrónico: milagriscelis@upei.edu.pe

\section{REFERENCIAS BIBLIOGRÁFICAS}

Alarcón,V., Alarcón, E., Figueroa,C., Mendoza, A. (2015). Tuberculosis en el Perú: situación epidemiológica, avances y desafíos para su control. Revista Peruana de Medicina Experimental y Salud Publica, 34 , 299-310. Recuperado de: https://doi.org/10.7861/ clinmedicin e.16-6-s79.

Bélanger, P. (1997). Educación para la salud. Hamburgo: Ediciones Druckerei Seemann. Recuperado de http://www.unesco.org/ education/u ie.

Bonilla, C. (2008). Situación de la tuberculosis en el Perú. Acta Médica Peruana, 25(3), 163 - 170. Recuperado de https:// mail-attachment.googleusercontent. com/attachment/u/0/?ui=2\&ik =71029 $2 \mathrm{ad} 8 \mathrm{a} \& \mathrm{view}=\mathrm{att} \& \mathrm{th}=160 \mathrm{c} 6 \mathrm{c} 43 \mathrm{f} 64$ 485 e $6 \&$ attid $=0.1 \&$ dis $p=i n l i n e \& r e a l$ attid=f_jcorr9da0\&safe $=1 \& z w \& s a d$ $\mathrm{d} b a t=A^{-} \mathrm{NGjdJ} 8 \mathrm{uU} 5 \mathrm{CQCbTexe7} 7_{\text {_j }}$ $29 \mathrm{k} 5 \mathrm{kmZCfZKZ8QNMj93caiqkfCzur-}$ 7vSWExe3t_xwKOYy84HLPYCctjh FLJU.

Campo, A., y Oviedo, H. (2008). Propiedades psicométricas de una escala de la consistencia interna. Revista de Salud Pública, 10(5), 831839.

Choque, R. (2011). Promoción de prácticas saludables para profesores de educación secundaria de Ciencia, Tecnología y Ambiente en Salud Respiratoria. Recuperado de: http://blog. pucp.edu.pe/blog/raulch oque/wp- content/ uploads/sites/905/2016/04/TBC.pdf.

Contreras, G., Contreras, C., Jiménez, F., Solís, M., y Torres, C. (2004). Promoción de la Salud y Prevención de la Tuberculosis en Centros Penitenciarios. Recuperado de: http://www.faviolajimenez.com/wp- content/ uploads/2012/08/004_ma nual_tbc_pdf.pdf.

Crespo,C.,Huayapa.,J., y Marca, R. (2014). Efectividad del programa educativo respira sano en el nivel de conocimiento sobre la tuberculosis pulmonar en los estudiantes del $3^{\circ}$ al $5^{\circ}$ de secundaria; de la institución educativa corazones marianos del norte, puente piedra, lima, 2014 (tesis de pregrado). Universidad Nacional del Callao, Lima, Perú. Recuperado de: http://repositorio. unac.edu.pe/bitst ream/handle/UNAC/170/ Catherine Nérida_Tesis_Títuloprofesional_2 014. pdf? sequence $=1$ \&isAllowed $=y$

Factor, E. (2013). Relación entre el nivel de conocimiento y actitud sobre medidas preventivas frente a la tuberculosis pulmonar en escolares del nivel segundarlo de la institución educativa Mánuel A. Odria del distrito de ciudad Nueva Tacna, 2012 (tesis de pregrado). Universidad nacional jorge basad re grohmann, Tacna, Perú. Recuperado de: http://repositorio.unjbg.edu. pe/bitst ream/handle/UNJBG/445/TG0304. pdf?sequence=1\&isAllowed=y.

Farga, V. (2006). Hacia la erradicación de la tuberculosis. Revista Chilena de Enfermedades Respiratorias, 22, 55-67. Recuperado de: http:// www.scielo.cl/pdf/rcher/v22n1/art09.pdf.

Fuentes, J. (2009). Modelo de cambio conductual orientado a la promoción de estilos de vida saludable en la organización (tesis pregrado). Universidad de Chile, Santiago, Chile. Recuperado de: http://repositorio.uchile.cl/tesis/ uch ile/2009/cs-fuentes_j/pdfAmont/cs- fuentes _.j. pdf.

Gutiérrez, F., Gutiérrez, K., y Toledo, F. (2005). Factores asociados a la incidencia de tuberculosis en personas de 15 a 49 años residentes en los distritos V, VI y municipio de Tipitapa, 2004 (tesis porgrado). Universidad nacional autónoma de nicaragua, Managua, Nicaragua. Recuperado de: http://repositorio.unan.edu.ni/6440/1/t370.pdf.

Gutiérrez,D.,Moreno,C., y Gonzáles, L. (2010). Estudio del niño en contacto con paciente tuberculoso. Revista Chilena de Infectología , 27(5), 423-428. Recuperado de: https://scielo. conicyt.cl/pdf/rci/v27n 5/art09.pdf.

Herrera, A. (2009). Las estrategias de aprendizaje. Revista de Innovación y Experiencias Educativas, 45(6), Recuperado de http://prepajocotepec. sems.udg.m x/sites/default/files/estrategias_her rera_capita.pdf.

Lima, M., Neves, S., y Lima, J. . (2012). Actitudes de adolescentes hacia la salud: evaluación de un programa escolar de promoción de la salud en Sevilla, España. Revista de Salud Colectiva sevilla, 8(1), 47-

Recuperado de: http://www.redalyc.org/articulo. oa? id=73123964005.

Lozano, J., Plasencia, C., Ramos, D., García, R.y Mahíquez, L.(2009). Factores de riesgo socioeconómicos de la tuberculosis pulmonar en el municipio de Santiago de Cuba. Revista Médica 
Efectividad del programa educativo "Mi amigo y yo, unidos contra la TB" en la prevención de tuberculosis a estudiantes de una institución educativa pública, Ate- Vitarte, Lima

de Santiago de Cuba, 20 (12), 1-8. Recuperado de: http://scielo.sld.cu/pdf/san/v13n4/s an07109. pdf.

Mendieta, F., Mena, L., y Mendoza, J. (2012). Roll de la enfermera comunitaria (tesis de pregrado). Universidad Politecnica de Nicaragua, Managua, Nicaragua. Recuperado de: http:// repositorio.upoli.edu.ni/30/1/ Rol de la enfermeria comunitaria.pdf.

Morales, L., Ferreira, B., Oduardo, N., Pompa, Y., y Areas, I. (2015). Factores de riesgo asociados a la tuberculosis pulmonar, Bayamo, Granma, enero 2007 a diciembre 2012. Revista Médica Granma, 19(1), 1028-4818. Recuperado de: http://www.medigraphic.com/pdfs/ multimed/ mul-2015/mul151e.pdf.

Oblitas, L. (2013). Psicología de la salud y Calidad de vida. México: Ediciones Plaza y Valdés. Recuperado de: http://desarrollohumanointegral. or g/resources/Conductas-y-estilos- de-vida.pdf

Organización Mundial de la Salud. (2014). Salud para los adolescentes del mundo. Ginebra, Suiza: Organización Mundial de la Salud. Recuperado de: www.who.int/adolescent/second- decade.

Organización Panamericana de la salud. (2016). Situación de la tuberculosis en las Americas. Recuperado de: http://www.paho.org/hq/ index.php ?option=com_topics\&view=article \&id=59\&Itemid $=40776$.

Sandoval, P. (10 de noviembre del 2016). Estos son los siete distritos con más casos de tuberculosis. El comercio. Recuperado de https://elcomercio. pe/lima/son- siete-distritos-casos-tuberculosis147629.

Pérez, N. (2007). La importancia de la promoción a la salud dentro de las escuelas de educación básica (tesis pregrado). Universidad Autonoma del Estado de Hidalgo, Pachuca,México. Recuperado por: http://repository.uaeh.edu.mx/ bitst ream/bitstream/handle/123456789/10817/ La importancia de la promocion a I a salud.pdf?sequence=1.

Risueño, J. (2010). Hábitos deportivos y estilos de vida de los escolares en los municipios de la provincia de Almería. Revista Nuevas tendencias en Educación Física, Deporte y Recreación. 17, 49-53. Recuperado de: http://www.redalyc.org/ pdf/3457/3 45732283010.pdf.

Robles, M. (2013). Tuberculosis en menores de 15 años en el hospital Hipólito Unanue de Tacna. Revista Médica Basadrina, 7(2), 64. Recuperado de: http://repositorio.unjbg.edu.pe/bitst ream/ handle/UNJBG/1048/TM140_Robles_Mejia_ MM.pdf? sequence=1\&isAllowed $=\mathrm{y}$.
Soto, M. y Chavez, A. (2015). Análisis de la situación epidemiológica de la tuberculosis en el Perú. Recuperado de: http://www2.congreso.gob. pe/sicr/ cendocbib/con4_uibd.nsf/693981 DC3C9D9765052580D6005ĀC863/\$FILE/1 asistbc.pdf.

Subirana, K. (23 de agosto de 2011). No hay distrito de Lima que se encuentre libre de la tuberculosis. El Comercio. Recuperado de: http://archivo. elcomercio.pe/socie dad/lima/no-hay-distritolima-que- se-encuentre-libre-tuberculosisnoticia-1149808.

Villar, M. (2011). Factores determinantes de la salud: Importancia de la prevención. Acta Médica Peruana, 28(4), 237-241. Recuperado d e : http://www.scielo.org.pe/pdf/amp/v 28n4/a11.pdf.

Vincezi, A. y Tedesco, F. (2009). La educación como proceso de mejoramiento de la calidad de vida de los individuos y de la comunidad. Revista Iberoamericana de Educación, 49(7), 1-12 Recuperado de: https://dialnet.unirioja. es/servlet/ar ticulo?codigo=3116567\&info=resu men\&idioma=SPA.

Wilches, E., Hernández, N., Hernández, O., \& Pérez, C. (2016). Conocimientos, actitudes, prácticas y educación sobre tuberculosis en estudiantes de una facultad de salud. Revista de Salud Pública, 18(1), 129-14.https://doi.org/10.15446/rsap.v18n 1.42424

Recibido: 07/04/18 Aceptado: 19/09/18 